\title{
Evaluation of the vascular protective effects of new oral anticoagulants in high-risk patients with atrial fibrillation (PREFER-AF): study protocol for a randomized controlled trial
}

Jin-Bae Kim, Hyun Jun Joung, Jung Myung Lee, Jong Shin Woo, Woo-shik Kim, Kwon Sam Kim, Kyung Hye Lee and Weon Kim ${ }^{*}$

\begin{abstract}
Background: Atrial fibrillation (AF) is known to be associated with several pathophysiological mechanisms including endothelial dysfunction of the heart and arterial vessels. Recent evidence suggests that new oral anticoagulant (NOAC) treatment may improve endothelial function and the inflammatory process involved in atherosclerosis in AF patients. This study is designed to determine the efficacy of NOAC therapy in the prevention of endothelial dysfunction and the progression of atherosclerosis of AF subjects.

Method/design: AF patients with a CHA2DS2-VASC score $>2$ and no previous history of overt coronary disease, severe peripheral arterial disease (PAD) or major stroke will be registered and randomly assigned either to the NOAC group (dabigatran or rivaroxaban) or the warfarin group in this prospective, randomized, 2-year follow-up study. Reactive hyperemia peripheral arterial tonometry (RH-PAT) measurements reflecting endothelial function will be conducted using the Endo-PAT2000 device. Left and right carotid intima-media thickness (IMT) will be measured at baseline, 12 months, and 24 months. The primary endpoint is defined as change in Reactive Hyperemia Index $(\mathrm{RHI})$ at 12 months. Secondary endpoints included changes in the right and left maximum IMT of the common carotid artery (CCA) and internal carotid artery (ICA), the mean IMT of the CCA and ICA at 24 months, and 24month cardiovascular events including cardiac death, stroke, acute myocardial infarction (AMI), overall cause of death, withdrawal of drug, or bleeding events.
\end{abstract}

Discussion: This is the first study to evaluate the efficacy of NOAC therapy for the prevention of endothelial dysfunction and progression of atherosclerosis in AF subjects. These findings are expected to expand the knowledge of NOAC pleotropic action in AF patients.

Trial registration: ClinicalTrials.gov: NCT02544932, registered on 7 September 2015.

Keywords: Atrial fibrillation, Anticoagulant, Atherosclerosis, Endothelium

Abbreviations: AF, Atrial fibrillation; AMI, Acute myocardial infarction; CCA, Common carotid artery; FMD, Flowmediated vasodilatation; ICA, Internal carotid artery; IMT, Intima-media thickness; IRB, Institutional Review Board; NOAC, New oral anticoagulants; PAR, Protease-activated receptor; PREFER-AF, PREvention of thromboembolic events - European Registry in Atrial Fibrillation; RHI, Reactive Hyperemia Index; RH-PAT, Reactive hyperemia peripheral arterial tonometry

\footnotetext{
* Correspondence: mylovekw@hanmail.net

Division of Cardiology, Department of Internal Medicine, Kyung Hee

University Hospital, Kyung Hee University, Seoul, Hoegi-dong 1,

Dongdaemun-gu, Seoul 130-701, Republic of Korea
} 


\section{Background}

Factor Xa and thrombin are well-known components of the coagulation cascade and other biological and pathophysiological processes that are known targets for effective anticoagulation treatment [1]. Therefore, the properties of oral, direct inhibitors of factor Xa (e.g., rivaroxaban) and thrombin (e.g., dabigatran) have been examined for hemostasis and thromboembolism management.

Accumulating evidence suggests that factor $\mathrm{Xa}$ and thrombin are important modulators of other cellular signaling mechanisms through the activation of proteaseactivated receptor (PAR)-mediated signaling. Cross-talk between coagulation and inflammatory pathways via thrombin- or factor Xa-mediated PAR activation on the arterial vessel wall and heart, and the resulting contribution to atherosclerosis and atrial fibrillation (AF), have been well-documented [2]. The endothelium, platelets, pro-inflammatory cytokines and chemokines, and several serine proteases (e.g., tissue factor (TF), factor Xa, thrombin), via the activation of PARs, are major points in for the promotion of inflammation and leukocyte migration, which results in the initiation of atherosclerosis [1-4].

Preclinical studies have provided evidence for the effects of direct Xa or thrombin inhibition beyond anticoagulation, including anti-inflammatory and protective activities in atherosclerotic plaque development [5-8]. Evidence has demonstrated that direct thrombin inhibition impairs the formation and size of atherosclerotic plaques in addition to preventing the progression of endothelial injury-associated stenosis in an apolipoprotein E-deficient mouse model [5, 6]. Also, factor Xa inhibition was shown to ensure the reduction of restenosis after balloon angioplasty of atherosclerotic femoral arteries in rabbits [7].

However, questions remain regarding the treatment concentration and adverse effects of these drugs. Furthermore, there is little evidence of similar effects in patients with AF. Therefore, this study will evaluate the protective effects of NOAC with reactive hyperemia peripheral arterial tonometry (RH-PAT) measurements made using the Endo-PAT2000 device, reflecting endothelial function and intima-media thickness (IMT) of the common carotid artery (CCA), which is used as a surrogate endpoint of atherosclerosis. In this study, we plan to analyze the occurrence of cardiovascular events in AF patients who are at high risk of these.

\section{Method/design}

\section{Study subjects and hypothesis}

Rivaroxaban and dabigatran have demonstrated potential for the management of thromboembolic disorders such as AF or deep vein thrombosis with or without pulmonary thromboembolism $[9,10]$. We therefore plan to initiate a prospective, randomized, 2-year follow-up study to clarify the efficacy of rivaroxaban and dabigatran in affecting change of endothelial function and progression of atherosclerosis in AF patients. The working hypothesis of this trial is that rivaroxaban and dabigatran are superior to standard vitamin $\mathrm{K}$ (vitK)-antagonists in the prevention of endothelial dysfunction, assessed by peripheral arterial tone (PAT) ratio at 12 months, and the progression of CCA-IMT at 12-month and 24-month follow-up.

\section{Study design}

This study is a prospective, randomized, 2-year followup study to clarify the efficacy of rivaroxaban and dabigatran in change of endothelial function and progression of atherosclerosis in AF patients. The study algorithm is shown in Fig. 1. After enrollment, subjects will be randomly assigned to the dabigatran group (110 mg or $150 \mathrm{mg}$ twice/day; group 1) or the rivaroxaban group (20 mg/day; group 2) or the warfarin group (controlled by international normalized ratio (INR) of $2-3$; group 3 ). After participation in the study procedure, clinical follow-up will occur at 1, 3, 12, and 24 months. Followup will be conducted via telephone interviews or office visits. Any adverse reactions will be assessed. This study is an investigator-initiated clinical trial with grant support from the Ministry of Health, Welfare, and Family Affairs of the Republic of Korea. The authors are solely responsible for the design, conduct, analysis, and manuscript preparation for this study. The 2-year duration of the study period will have a registration period from September 2015 to February 2016, with complete study duration from September 2015 to April 2018.

\section{Study population}

Patients aged between 40 to 85 years will be enrolled in the study if they have been diagnosed with AF and have a $\mathrm{CHA}_{2} \mathrm{DS}_{2}$-VASc score above 2 points (Table 1 ). The following exclusion criteria have been set: severe peripheral arterial disease (PAD) (greater than a Fontaine IIb category), grade-4 or higher cerebral infarction on the modified Rankin Scale, and proven coronary artery disease based on coronary angiogram. In addition, patients with a range of concomitant comorbidities including severe hepatic or renal dysfunction, uncontrolled congestive heart failure, uncontrolled hypertension or diabetes mellitus, hematological disorders, or those with allergy or hypersensitivity to the investigational drugs, pregnant or lactating women, or women wishing to become pregnant, will be excluded. Written informed consent will be obtained from all patients, and the Ethics Review Board of Kyung Hee University Hospital will review the study.

\section{Randomization and allocation concealment}

Randomization will be performed using a computergenerated random number list with a permuted block 


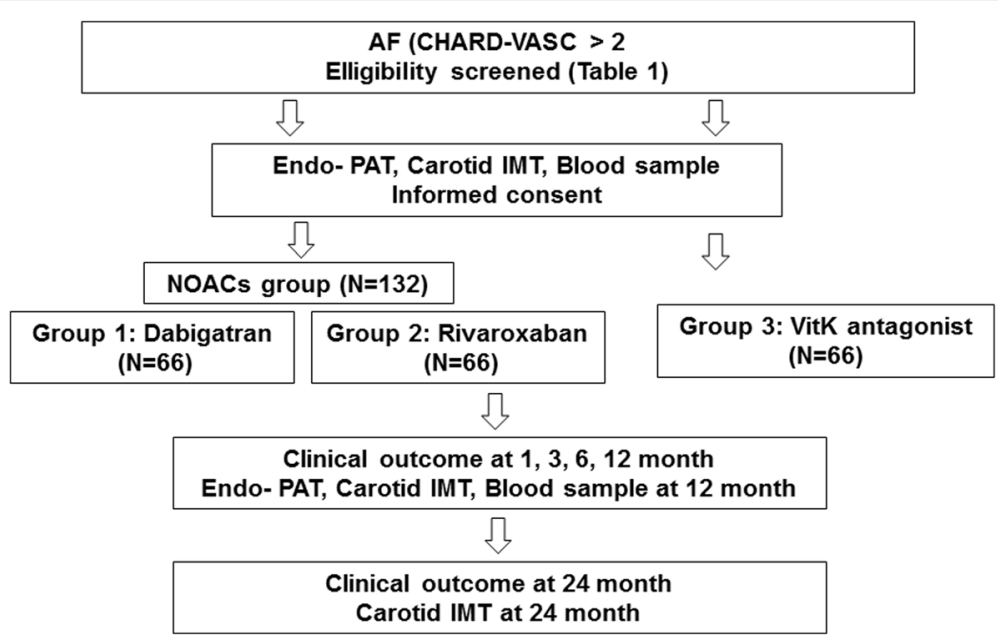

Fig. 1 PREFER-AF trial flow diagram. Participants will receive new oral anticoagulant (NOAC) or vitamin K (vitK)-antagonist treatment according to the assigned sequence; then, endothelial function and clinical events will be assessed

design at a 1:1:1 ratio. Adequate allocation blinding will be ensured by the following procedure. After obtaining participant consent, allocation information will be sent to the main investigator at Kyung Hee University hospital by telephone, and allocations will not be known by the participants or enrolling investigators.

\section{Outcome measures}

The primary endpoint is defined as change in the Reactive Hyperemia Index (RHI) at 12 months. Secondary

Table 1 Enrollment criteria

Inclusion criteria

1. $\mathrm{AF}\left(\mathrm{CHA}_{2} \mathrm{DS}_{2}\right.$-VASc score $>2$ points $)$

2. Aged between 40 and 85 years

Exclusion criteria

1. Aged below 40 or over 85 years

2. History of myocardial infarction

3. Proven coronary artery disease by coronary angiogram

4. Cerebrovascular disorders rated as grade 4 or greater on the modified Rankin Scale

5. Severe peripheral arterial disease rated as Fontaine llb or over

6. Uncontrolled hypertension $(180 / 110 \mathrm{mmHg})$ or diabetes (glycated hemoglobin: HbA1C >10.0\%)

7. Severe hepatic dysfunction (aspartate transaminase (AST)/alanine transaminase (ALT) $>100 \mathrm{mg} / \mathrm{dL}$ )

8. Renal dysfunction (serum creatinine $\geq 2.0 \mathrm{mg} / \mathrm{dL}$ )

9. Uncontrolled congestive heart failure

10. Severe hematological abnormalities

11. Drug allergies or history of hypersensitivity to the investigational drugs

12. Pregnant or lactating women, or women who wish to become pregnant endpoints included changes in the right and left maximum IMT of the CCA and internal carotid artery (ICA), mean IMT of the CCA and ICA at 24 months, and 24month cardiovascular events including cardiac death, stroke, acute myocardial infarction (AMI), overall cause of death, withdrawal of drug, or bleeding events.

\section{Procedures}

Measurement of PAT ratio will be performed with the standard technique and device [11-14]. Endo-PAT measurements will be performed at baseline and at 12 months after randomization. The RH-PAT 2000 device (Itamar Medical, Caesarea, Israel) will be used for digital RHPAT to evaluate endothelial function as previously described. The RH-PAT Index (RHI) reflects the extent of reactive hyperemia and is calculated as the ratio of the average amplitude of PAT signal over 1 min starting 1.5 min after cuff deflation (control arm, A; occluded arm, C) divided by the average amplitude of PAT signal at the 2.5-min time period before cuff inflation (baseline) (control arm, B; occluded arm, D). Thus:

$$
R H I=(C / D) /(A / B) \times \text { baseline correction. }
$$

Impaired endothelial function is defined as a log RHI less than 0.6, and favorable endothelial function is defined as a log RHI more than or equal to 0.6 .

Measurement of CCA-IMT will be performed with the standard technique and device [12, 15]. Imaging studies of the left and right carotid arteries will be performed by expert sonographers who are well-trained in the use of $10-\mathrm{MHz}$ linear vascular probes (Vivid 7, GE Vingmed Ultrasound, Horten, Norway). Measurements will be performed at baseline, and at 12 months and 24 months after randomization. To avoid interobserver variability, 
the same investigator will examine each participant with the same equipment throughout all the visits. The IMT will be measured as the distance between two parallel echogenic lines corresponding to the blood-intima and media-adventitia interface on the posterior wall of the artery. Three IMT determinations will be performed at the site of the thickest point with a maximum CCAIMT and two adjacent points $(1 \mathrm{~cm}$ upstream and $1 \mathrm{~cm}$ downstream from this site). These three determinations will be averaged (mean CCA-IMT). The CCA is defined as the segment extending from 10 to $20 \mathrm{~mm}$ proximal to the tip of the common carotid bifurcation site. The ICA is defined as the segment $10 \mathrm{~mm}$ distal to the tip of the common carotid bifurcation site, and the bifurcation is defined as the segment between the CCA and the ICA. Carotid IMT will be measured using dedicated software (Intimascope, Media Cross Co., Tokyo, Japan) by an examiner blinded to all clinical information.

Blood samples will be obtained from subjects in the fasting state. The von Willebrand factor, interleukin (IL)-10, and adiponectin endothelial cell markers, the IL-6, tumor necrosis factor alpha (TNF- $\alpha$ ), and highsensitivity C-reactive protein (hs-CRP) inflammatory markers, the vascular adhesion molecule and ICAM-1 adhesion molecules, and the CD-40 ligand, P-selectin, and cathepsin-K platelet activation markers will be measured using standard methods.

\section{Sample size measure and statistics}

This study is a prospective, pilot study for a proof of concept trial. In a previous studies, the RHI was $1.63 \pm 0.28$ in patients with permanent/persistent $\mathrm{AF}$ $[13,14]$. We assume that NOAC will improve RHI values by an $8 \%$ difference with no significant difference between the two different NOACs. The expected difference in RHI was driven from clinical significances and previous medical treatments in other study population [16-18]. To detect a statistically significant difference with a power of $80 \%$ with a two-sided $\alpha$-level of 0.05 , a sample of 165 patients (55 patients for each group) will be required. Assuming dropout rates would be $20 \%$, the total sample size will be set at 198 subjects.

Statistical analyses will be carried out using the SPSS Statistics for Windows ver. 17.0 software package (SPSS Inc., Chicago, IL, USA). A two-sided $p<0.05$ is considered significant. Continuous variables, presented as means \pm standard deviations, will be evaluated for normal distribution and compared using the Student $t$ test or analysis of variance test accordingly. The continuous parameters with a skewed distribution will be logarithmically transformed and categorical variables, presented as frequencies and percentages, will be compared using the chi-square test or Fisher's exact test when appropriate.
Repeated measures parameters over time between the groups will be determined by repeated measure analysis of variance with an autoregressive model or analysis of covariance adjusting for baseline values. The clinical event rates will be assessed using the Kaplan-Meier method and the log-rank test will be used to compare survival between the two groups and the Cox proportional hazards model will be used for multivariate analysis (forward stepwise) to investigate which of the parameters identified using univariate analysis are significantly associated with clinical events.

\section{Discussion}

The aim of this study is to determine the efficacy of NOAC therapy in the prevention of endothelial dysfunction and progression of atherosclerosis in AF subjects. Recent evidence suggests that AF adversely affects endothelial function $[11,13,14]$. Further, conversion of AF to sinus rhythm results in improved flow-mediated vasodilatation (FMD) in patients with persistent AF $[13,14]$. It is not known whether the vitK antagonists are effective in affecting endothelial function and atherosclerotic progression in AF patients. Preclinical evidence shows that NOACs are effective in reducing inflammatory cytokines and atherosclerotic cascades [5-8]; however, clinical data are lacking. We believe that the therapeutic effects resulting from aspirin or warfarin and NOACs, which have different mechanisms of action, have not been sufficiently investigated in AF patients. The results of this study are expected to broaden our understanding of NOAC mechanisms in humans and lead to the development of appropriate drug therapies for patients with AF and atherosclerosis. This is a very important objective because the incidence of AF and its common complications are rising at a staggering rate in many countries.

One of the study limitations is that the primary outcome in this study is not a hard endpoint for clinical outcome but a surrogate marker for endothelial dysfunction. Therefore, further study for clinical outcome of vascular events comparing NOACs and warfarin should follow after this trial. Furthermore, this study is not double-blind trial. To minimize the bias, the technician performing examination and analysis will be blind to the allocation of patients.

\section{Trial status}

This study is ongoing. We are currently recruiting and enrolling subjects at the Kyung Hee University Medical Center.

\section{Acknowledgement}

This study was supported by a grant from the Traditional Korean Medicine R\&D Project, Ministry of Health and Welfare, Republic of Korea (HI15C0166). 


\section{Authors' contributions}

JB Kim participated in the design and coordination and drafted the atrial fibrillation study protocol as first author. HJ Joung participated in the design and coordination and drafted the atrial fibrillation study protocol as co-first author. JM Lee participated in the design of the study and will perform statistical analyses. JS Woo participated in the design of the study and will perform statistical analyses. WS Kim will help to draft the manuscript and carry out noninvasive imaging studies. KS Kim participated in the design and coordination and will direct the manuscript. KH Lee participated in the design and coordination and will help to evaluate this project as a multidisciplinary project. W Kim participated in the design and will direct and draft the manuscript as a corresponding author. All authors read and approved the final manuscript.

\section{Competing interests}

The authors declare that they have no competing interests.

\section{Ethics approval and consent to participate}

The Institutional Review Board at Kyung Hee University Hospital approved this study (KMC IRB 1537-06) and the protocol for the trial has been registered at https://clinicaltrials.gov/ct2/show/NCT02544932 (NCT 02544932).

Received: 20 January 2016 Accepted: 5 August 2016

Published online: 24 August 2016

\section{References}

1. Eriksson BI, Quinlan DJ, Eikelboom JW. Novel oral factor Xa and thrombin inhibitors in the management of thromboembolism. Annu Rev Med. 2011;62:41-57.

2. Borissoff Jl, Spronk HM, ten Cate H. The hemostatic system as a modulator of atherosclerosis. N Engl J Med. 2011;364(18):1746-60.

3. Spronk HM, de Jong AM, Crijns HJ, Schotten U, Van Gelder IC, Ten Cate H. Pleiotropic effects of factor $\mathrm{Xa}$ and thrombin: what to expect from novel anticoagulants. Cardiovasc Res. 2014;101(3):344-51.

4. Esmon CT. Targeting factor $\mathrm{Xa}$ and thrombin: impact on coagulation and beyond. Thromb Haemost. 2014;111(4):625-33.

5. Lee IO, Kratz MT, Schirmer SH, Baumhakel M, Bohm M. The effects of direct thrombin inhibition with dabigatran on plaque formation and endothelial function in apolipoprotein E-deficient mice. J Pharmacol Exp Ther. 2012;343(2):253-7.

6. Borissoff Jl, Otten JJ, Heeneman S, Leenders P, van Oerle R, Soehnlein O, et al. Genetic and pharmacological modifications of thrombin formation in apolipoprotein e-deficient mice determine atherosclerosis severity and atherothrombosis onset in a neutrophil-dependent manner. PLoS One. 2013;8(2), e55784.

7. Ragosta M, Gimple LW, Gertz SD, Dunwiddie CT, Vlasuk GP, Haber HL, et al. Specific factor $\mathrm{Xa}$ inhibition reduces restenosis after balloon angioplasty of atherosclerotic femoral arteries in rabbits. Circulation. 1994;89(3):1262-71.

8. Borissoff Jl, Heeneman S, Kilinc E, Kassak P, Van Oerle R, Winckers K, et al Early atherosclerosis exhibits an enhanced procoagulant state. Circulation. 2010;122(8):821-30.

9. Patel MR, Mahaffey KW, Garg J, Pan G, Singer DE, Hacke W, et al. Rivaroxaban versus warfarin in nonvalvular atrial fibrillation. N Engl J Med. 2011;365(10):883-91.

10. Connolly SJ, Ezekowitz MD, Yusuf S, Eikelboom J, Oldgren J, Parekh A, et al. Dabigatran versus warfarin in patients with atrial fibrillation. N Engl J Med. 2009;361(12):1139-51.

11. Wong CX, Lim HS, Schultz CD, Sanders P, Worthley MI, Willoughby SR Assessment of endothelial function in atrial fibrillation: utility of peripheral arterial tonometry. Clin Exp Pharmacol Physiol. 2012;39(2):141-4.

12. Woo JS, Jang WS, Kim HS, Lee JH, Choi EY, Kim JB, et al. Comparison of peripheral arterial tonometry and flow-mediated vasodilation for assessment of the severity and complexity of coronary artery disease. Coron Artery Dis. 2014;25(5):421-6.

13. Skalidis El, Zacharis EA, Tsetis DK, Pagonidis K, Chlouverakis G, Yarmenitis S, et al. Endothelial cell function during atrial fibrillation and after restoration of sinus rhythm. Am J Cardiol. 2007;99(9):1258-62.

14. Yoshino S, Yoshikawa A, Hamasaki S, Ishida S, Oketani N, Saihara K, et al. Atrial fibrillation-induced endothelial dysfunction improves after restoration of sinus rhythm. Int J Cardiol. 2013;168(2):1280-5.
15. Katakami N, Kim YS, Kawamori R, Yamasaki Y. The phosphodiesterase inhibitor cilostazol induces regression of carotid atherosclerosis in subjects with type 2 diabetes mellitus: principal results of the Diabetic Atherosclerosis Prevention by Cilostazol (DAPC) study: a randomized trial. Circulation. 2010;121(23):2584-91.

16. Clarkson P, Montgomery HE, Mullen MJ, Donald AE, Powe AJ, Bull T, et al. Exercise training enhances endothelial function in young men. J Am Coll Cardiol. 1999;33(5):1379-85.

17. Flammer AJ, Hermann F, Wiesli P, Schwegler B, Chenevard R, Hürlimann D, et al. Effect of losartan, compared with atenolol, on endothelial function and oxidative stress in patients with type 2 diabetes and hypertension. J Hypertens. 2007;25(4):785-91.

18. Reriani MK, Dunlay SM, Gupta B, West CP, Rihal CS, Lerman LO, et al. Effects of statins on coronary and peripheral endothelial function in humans: a systematic review and meta-analysis of randomized controlled trials. Eur J Cardiovasc Prev Rehabil. 2011;18(5):704-16.

\section{Submit your next manuscript to BioMed Central and we will help you at every step:}

- We accept pre-submission inquiries

- Our selector tool helps you to find the most relevant journal

- We provide round the clock customer support

- Convenient online submission

- Thorough peer review

- Inclusion in PubMed and all major indexing services

- Maximum visibility for your research

Submit your manuscript at www.biomedcentral.com/submit
Biomed Central 